\title{
RÉSUMÉ
}

Le but de ce rapport est de montrer que l'approche biogéochimique constitue un cadre unitaire pour gérer au mieux l'environnement de la planète et en particulier de la planète anthropisée.

Ce cadre repose sur l'étude des cycles biogéochimiques qui caractérisent la biosphère (c'est-à-dire là où se manifeste la vie), et qui sont naturellement plus ou moins liés aux cycles géochimiques généraux du Globe terrestre.

Ceci nous conduit à préciser les limites du rapport.

1. Le rapport ne prend pas en compte toute la planète, mais seulement la partie supérieure où se déroulent les phénomènes géodynamiques externes, mais où peuvent intervenir aussi dans un certain nombre de situations des phénomènes de géodynamique interne (volcanisme...).

2. Dans cette partie supérieure, le rapport porte plus spécialement sur les zones continentales. Naturellement, les terres émergées et les océans sont liés, mais comme ces derniers à la surface du globe ne fonctionnent pas de la même façon, le travail s'adresse principalement aux écosystèmes terrestres.

3. Sur les continents, le rapport est focalisé sur la biosphère, qui constitue le système écologique.

Ce système écologique est situé entre :

- le système climatique, où le cycle de l'océan et de l'atmosphère est primordial ;

- le système géologique, qui correspond à la géosphère azoïque.

4. Dans le système écologique terrestre, la circulation des éléments fait appel à quatre sortes de relations :

- plante-atmosphère : sous-cycle atmosphérique;

- intérieur de la plante : sous-cycle biochimique;

- sol-plante : sous-cycle biologique;

- roche-sol : sous-cycle géochimique. 
Les cycles biogéochimiques reposent principalement sur les deux derniers sous-cycles où interviennent les sols qui sont de ce fait les pivots du fonctionnement de la biosphère terrestre.

La finalité de ce rapport n'était pas d'aborder les cycles biogéochimiques de tous les éléments chimiques, mais de montrer pourquoi et comment ces cycles sont amenés à jouer un grand rôle dans l'évolution de la planète anthropisée.

Pour ce faire, le rapport a été divisé en deux ensembles et quatre parties :

- Le premier ensemble regroupe tout ce qui a trait directement à la connaissance des cycles biogéochimiques. Il comporte deux parties.

La première partie concerne la description du cycle biogéochimique de quelques éléments qui jouent un rôle majeur dans la biogéosphère; ont été retenus :

- le carbone, du fait qu'il intervient dans tous les cycles : atmosphérique, écologique et géologique (chapitre 1);

- l'azote, le phosphore et le soufre parce qu'ils sont typiques du monde vivant et qu'ils sont susceptibles de jouer un rôle de premier ordre dans l'environnement (chapitre 2);

- le silicium, l'aluminium et le fer qui permettent de faire le pont entre le système écologique et le système géologique (chapitre 3);

- enfin, les radionucléides (naturels et artificiels), en raison de l'impact qu'ils peuvent avoir sur l'environnement biologique (chapitre 4).

la deuxième partie porte sur l'étude biogéochimique de quelques milieux représentatifs de la planète naturelle et anthropisée.

Dans les écosystèmes naturels sont abordés ainsi :

- les écosystèmes forestiers, qui sont très caractéristiques des milieux terrestres et qui sont le siège de recyclages biogéochimiques souvent très performants (chapitre 5.1);

- les milieux océaniques; la biogéochimie de ces milieux a été brièvement décrite, bien que ne faisant pas partie à proprement parler du sujet, pour bien montrer que dans ce domaine tout est lié à la surface du globe (chapitre 5.2). 
Dans les milieux à anthropisation accentuée, c'est-à-dire là où des excédents et des surcharges en certains éléments peuvent entraîner des dysfonctionnements, trois situations de ont été retenues :

- dans le domaine agricole est abordé le cas des épandages de matières organiques exogènes (MOE), que l'on rencontre de nos jours dans les bassins d'élevage intensif (chapitre 6.1);

- pour le secteur industriel sont présentés quelques milieux où certains éléments métalliques sont en excès, comme cela se produit dans les zones minières et métallurgiques (chapitre 6.2);

- enfin, les milieux estuariens, du fait que ceux-ci sont le réceptacle de nombreux contaminants en provenance des continents (chapitre 6.3).

- Le second ensemble résulte de tout ce que le premier nous a appris, à savoir que les cycles biogéochimiques sont la conséquence de systèmes complexes qui font appel à beaucoup de disciplines et nécessitent de nombreuses données; ce qui implique:

(a) un approfondissement dans certains secteurs scientifiques;

(b) un intérêt particulier pour la collecte des données;

(c) un recours aux travaux de modélisation.

Le point $(a)$ constitue la troisième partie, où on met l'accent sur le développement de tout ce qui concerne le rôle du milieu vivant des sols, domaine où les connaissances sont encore bien déficitaires; sur cette question deux points sont abordés en particulier :

- les recherches sur les micro-organismes qui sont à la base de la transformation des résidus des êtres vivants et de leur retour à l'état minéral (chapitre 7);

- les études sur les matières organiques des sols; celles-ci jouant en effet un rôle fondamental, du fait qu'elles sont intermédiaires entre le monde minéral et le monde vivant (chapitre 8).

Les points (b) et (c) sont l'objet de la quatrième partie qui traite des opérations de modélisation (chapitre 9), avant d'envisager le problème de la collecte générale des données (observatoires, réseaux, ...) (chapitre 10). 


\section{Conclusions et recommandations}

Elles concernent plus particulièrement les trois points suivants :

1. mettre l'accent à l'avenir sur un certain nombre de disciplines encore souvent orphelines; par exemple la chimie bio-inorganique, l'écologie microbienne, la science des sols, ... .

2. sortir, dans ce domaine de recherche, des disciplines classiques et prôner la multidisciplinarité; ce qui implique :

- la création de départements transversaux au sein des grands organismes,

- le développement des relations interorganismes par la mise en place d'unités mixtes,

- l'appui à réaliser dans le domaine des observatoires : création, suivi et surtout maintenance dans le long terme;

3. enfin, dans l'enseignement supérieur, envisager la création de structures universitaires permettant de former des étudiants qualifiés dans un secteur aussi crucial pour l'avenir de l'humanité. 